\title{
DESAFIOS DEL SECTOR TMT
}

América Latina se encuentra en un momento importante para la adopción de la economía digital. Según expertos, la tecnología se consolida cada vez más como un actor ideal dentro de los procesos productivos de cada país, es por esto que conocer los retos y tendencias del sector TMT nos permitirá ir un paso adelante y llevar estas soluciones a las diferentes industrias.

\section{TECNOLOGÍA}

Latinoamérica necesita soluciones de hardware y software: este año la inversión de la región se acercará a los \$275 MM y en los próximos tres años crecerá más de cinco veces.

La Inteligencia Artificial continúa desarrollándose en paralelo con la Nube y el Internet de las Cosas, lo que genera desconfianza sobre lo que las máquinas y las personas pueden hacer en conjunto.

\section{MEDIOS \& ENTRETENIMIENTO}

La música y el streaming tienen una baja inflación y la aparición de medios gratuitos o contenidos compartidos hacen que resulte especialmente complicado un crecimiento orgánico del sector.

5. La publicidad en TV crece de manera llamativa para los medios maduros, con mayor énfasis en TV paga y TV online, este último nuevo para el sector.

El entretenimiento mediante consolas y videojuegos sigue afectado por la piratería, los altos impuestos + :- a los dispositivos y el ilimitado ecosistema de ventas.

\section{TELECOMUNICACIONES}

La industria de las telecomunicaciones está trabajando en un estándar $\mathbf{5 G}$ que está se prevé estará terminado en 2020.

La única relación que la mayoría de los operadores de telecomunicaciones tiene con sus clientes es cuando hay un problema.

Las empresas de telecomunicaciones dependen de los ingresos por llamada y mensajes, sin embargo cada año son menos. 


\section{TENDENCIAS DEL SECTOR TMT}

La participación de EY en el GSMA Mobile World Congress 2017 fue una excelente oportunidad para mostrar nuestras capacidades en TMT, fortalecer nuestra marca globalmente y posicionarnos con clientes de esta industria. El evento reunió a más de 108.000 personas, incluyendo 5.500 líderes $\mathrm{C}$-suite del ecosistema TMT.
Como resultado de esta participación hemos consolidado las 6 tendencias clave que debemos tener en cuenta para el desarrollo de la economía digital.

\section{DISPOSITIVOS}

\section{※ LA INNOVACIÓN SE ESTÁ DESACELERANDO}
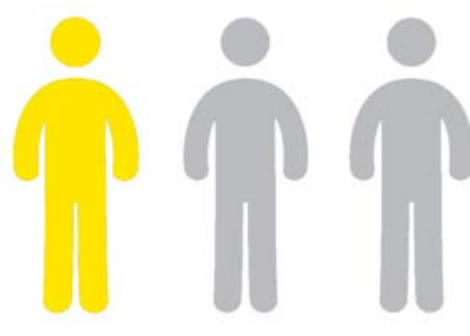

Uno de cada tres usuarios

adultos de internet están

buscando formas activas

para pasar el tiempo

desconectados.

Encuesta 2017 OFCOM Reino Unido.
El mercado móvil tiene un crecimiento más lento, los ciclos son más largos para el reemplazo de dispositivos y existe menos diferenciación.

\section{NUEVA TENDENCIA}

La innovación pasó a nuevas categorías de productos, como accesorios personales de realidad virtual (RV) y realidad aumentada (RA). Se espera que 20 millones de

\section{REALIDAD VIRTUAL}

La RV sumerge a los usuarios en mundos virtuales. Tiene millones de usuarios preparados y a la espera de sus últimos avances.

- Juegos de PCs y MMO (massively multiplayer online).

- Empresas desarrolladoras de consolas

- Distribuidores de cine para películas 3D, 4DX, IMAX.

- Investigación y desarrollo de aplicaciones para de fines militares, de salud o educativos. dispositivos de RV y RA se lancen este año. Las principales diferencias entre estos dispositivos son:

\section{REALIDAD AUMENTADA}

La RA incorpora objetos virtuales en el mundo real del usuario. Esta tecnología podría desempeñar un papel multisector. Las posibilidades que ofrecerá la RA incluyen:

- E-commerce y publicidad.

- Llamadas de voz.

- Navegación web.

- Apps empresariales o de consumo

- Juegos y atracciones de parques temáticos.

Fuentes: http://www.indracompany.com/es/blogneo/prometedores-mercados-realidad-virtual-realidad-aumentada https://www.idc.com/getdoc.jsp?containerld=prUS41676216 


\section{REDES MÓVILES 5G}

\section{* A MEDIDA QUE SE ACERCA LA REALIDAD, TAMBIÉN LO HACEN LOS OBTÁCULOS.}

\section{CONTEXTO:}

- Se necesita revolucionar la banda ancha móvil para habilitar el Internet de las cosas (IoT, por sus siglas en inglés)

- Existe gran discusión sobre cómo los principios de la nube pueden crear redes más ágiles que respondan a las necesidades del cliente, con menores costos de infraestructura.

- Lo operadores de telecomunicaciones tiene como prioridad responder a las soluciones comerciales esperadas con la implementación de la red 5G, la cual está prevista para el 2020.

\section{NUEVA TENDENCIA}

\section{Reformas Gubernamentales.}

Los gobiernos y entes reguladores aún tienen mucho trabajo por hacer, a medida que se habilitan nuevas bandas espectrales que puedan soportar la red $5 \mathrm{G}$.

\section{REDES EN CENTROAMÉRCA Y EL CARIBE}

Toda la región cuenta con $\mathbf{3 G}$ y $\mathbf{4 G}^{\mathbf{1}}$

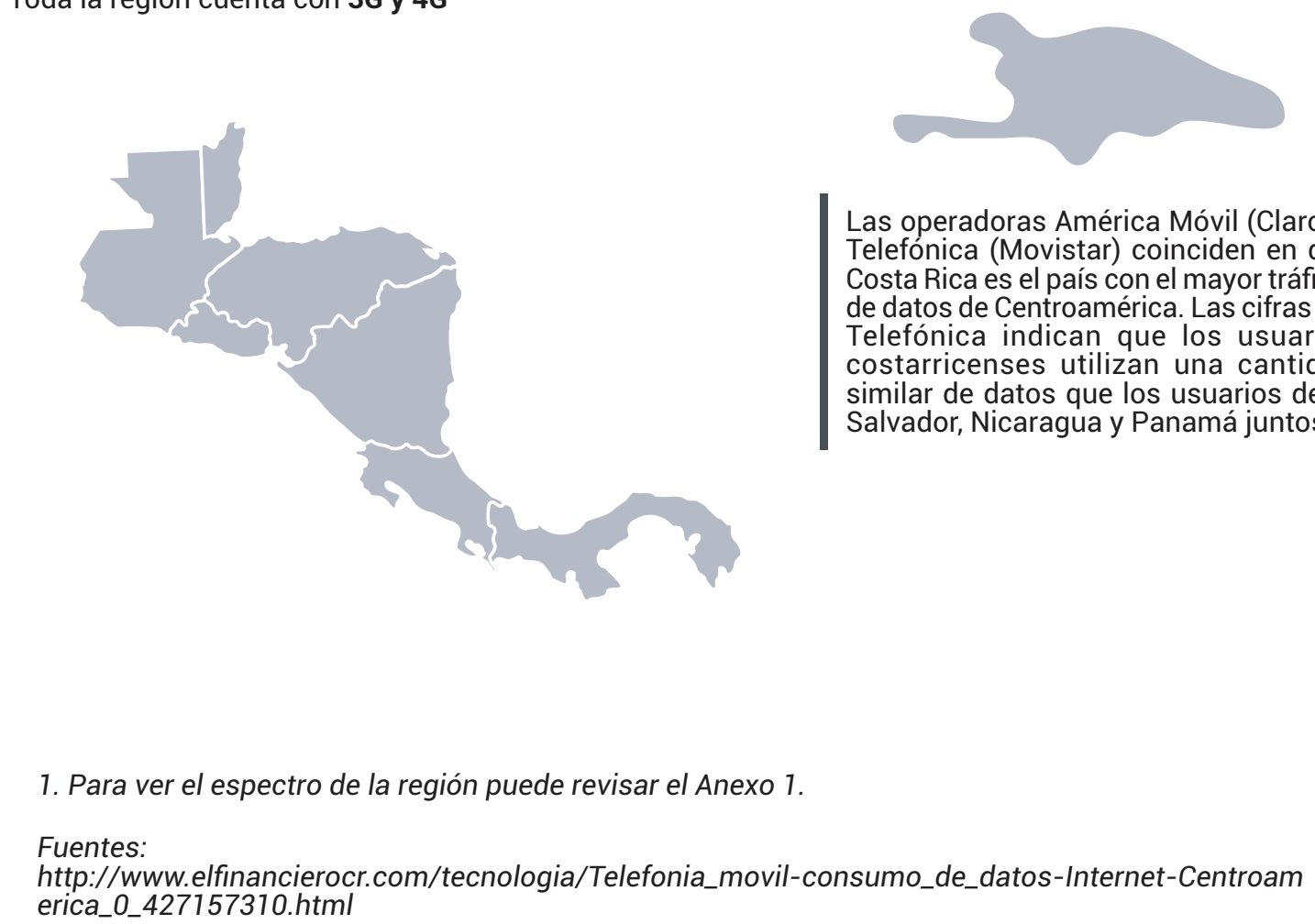

Estos deben considerar políticas más amplias para fomentar la inversión, de modo que la cooperación pública privada pueda liderar a la implementación de la economía digital 


\section{SOCIEDAD AÚN MÁS CONECTADA}

\section{$\%$ DESAFÍOS EN EL USO INDUSTRIAL DE LAS TECNOLOGÍAS.}

El interés comercial ha aumentado considerablemente en los últimos años particularmente en cuanto a los vehículos autónomos y hogares inteligentes. Por su parte los usuarios no se muestran del todo accesibles a arrancar con el uso de estas tecnologías, como lo demuestra la siguiente información extraída de la encuesta de Bundle Jungle.

\section{ENCUESTA SOBRE: HOGARES INTELIGENTES}

¿Cuál es la probabilidad de usar o comprar los siguientes productos inteligentes en los próximos cinco años?

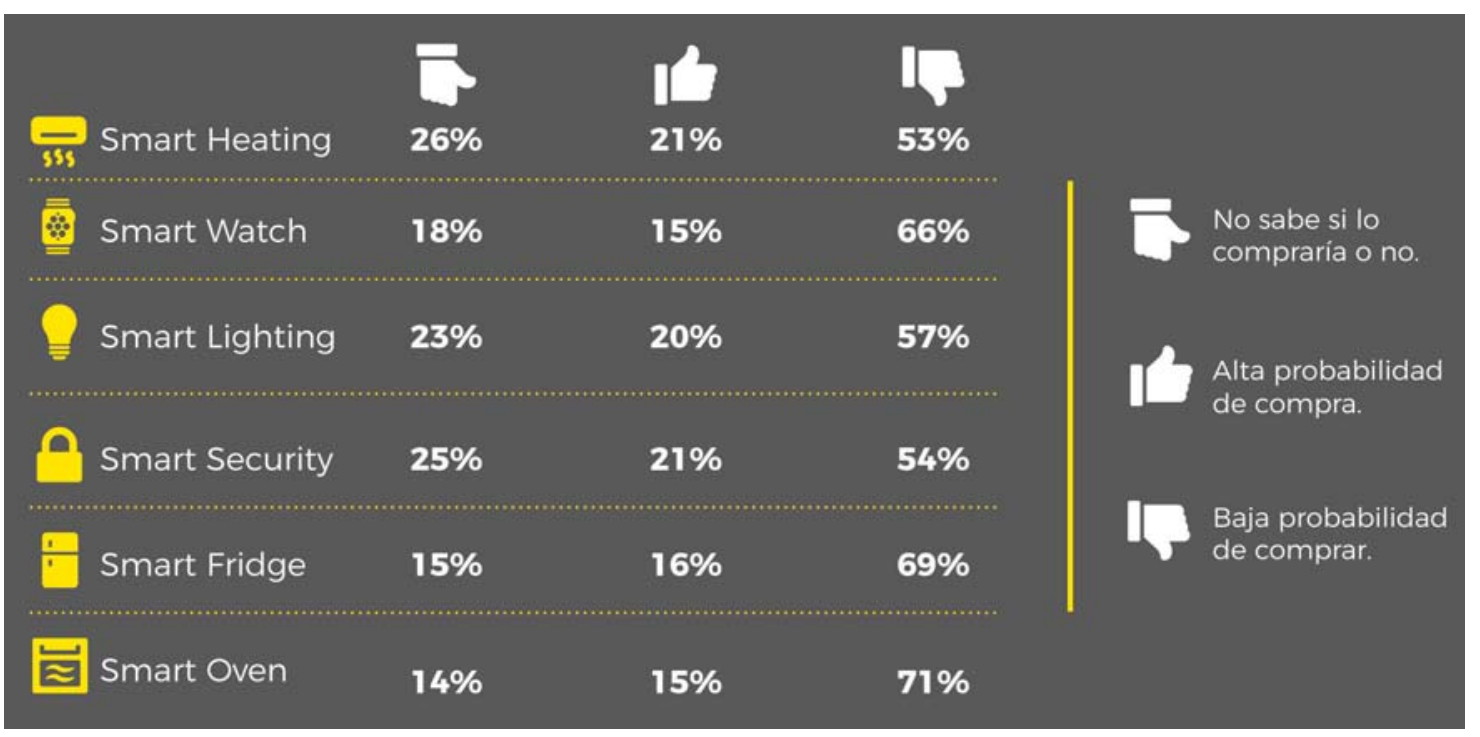

\section{NUEVA TENDENCIA}

Cientos de aplicaciones aisladas no satisfacen las expectativas de los usuarios. El crecimiento en el Internet de las cosas dependerá de las alianzas y la colaboración.
Los modelos de negocios deben trabajar en la interoperabilidad dado que las soluciones existentes están muy centradas en lo vertical.

Fuentes: Estudio Reino Unido. 2016. EY / Bundle Jungle 


\section{CONTEXTO:}

- Las compañías de telecomunicaciones han invertido años y miles de millones de dólares en la construcción de redes para abastecer los países.

- Los proveedores de televisión de pago están firmando acuerdos con los actores de OTT (Broadcasting).

- Los operadores móviles están afinando sus tarifas para permitirles a los usuarios de teléfonos inteligentes ver más contenido exclusivo.

\section{NUEVA TENDENCIA}

Alianzas con la "competencia", de cara a facturar lo que el usuario está utilizando las alianzas son la clave. Esto además es un buen augurio para el futuro del Internet de las cosas, donde hay mucho por trabajar pero no pueden ser esfuerzos aislados sino conjuntos.
Telco and OTT partnerships

2013-2016

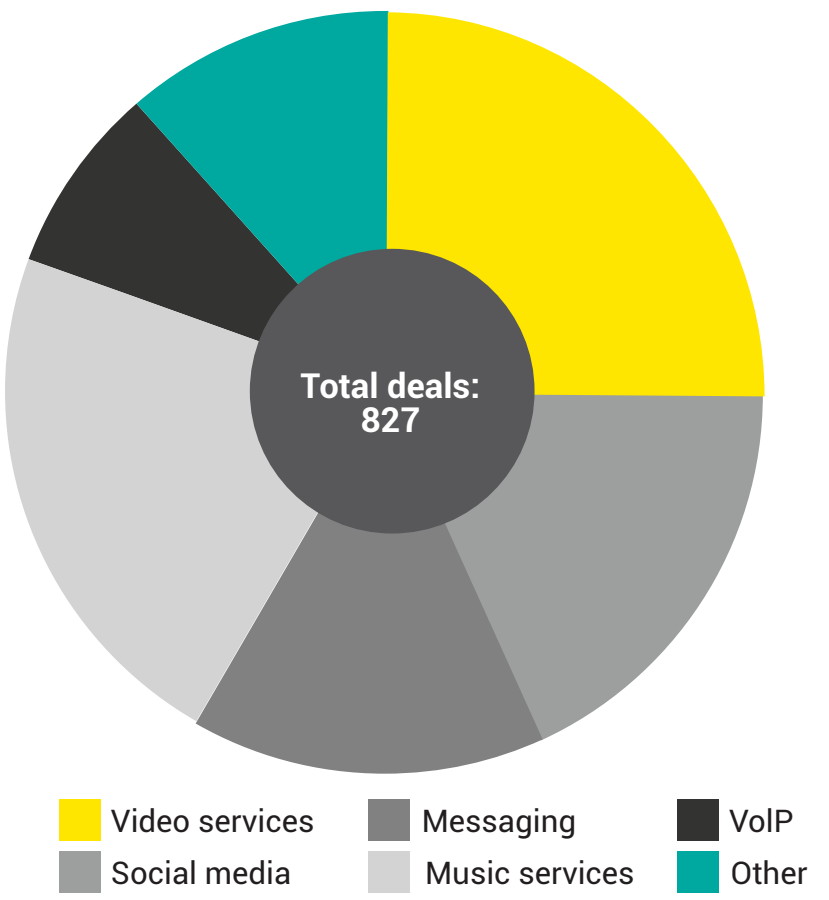

Los servicios Over-The-Top (OTT) son aquellos que se brindan a través de Internet, pero no necesitan elevadas inversiones ni requieren de infraestructura o espectro y no están sujetos al marco regulatorio de los operadores.

Entre estos servicios se encuentran las aplicaciones desarrolladas por startups, como Skype, Netflix, WhatsApp, las herramientas de búsqueda de Google, el correo web de Microsoft Hotmail, entre otras, es decir que para poder disfrutarlos existen solo dos requerimientos: contar con un dispositivo compatible y, lo más importante, una conexión a Internet.

Fuentes: http://mundocontact.com/plataformas-de-servicios-ott-la-cuarta-ola-tecnologica/ 


\section{ACERCARSE AL CLIENTE}

\section{\% LOS ASISTENTES DIGITALES E INTELIGENCIA ARTIFICIAL TOMAN PROTAGONISMO.}

\section{CONTEXTO:}

- La confusión de los clientes limita los niveles de lealtad e impide la adopción de nuevos productos y servicios.

- Hay un sentimiento creciente de que las compañías TMT pueden aprender de los actores en otros sectores, quienes tienen altos niveles de lealtad.

- Los chatbots y asistentes virtuales reflejan la importancia de nuevas experiencias del cliente, ya sea si se trata de personalizar los servicios consolidados o crear nuevos.

\section{NUEVA TENDENCIA}

De cara al futuro, nuevos avances en inteligencia artificial (IA) y aprendizaje automático determinarán el ritmo de

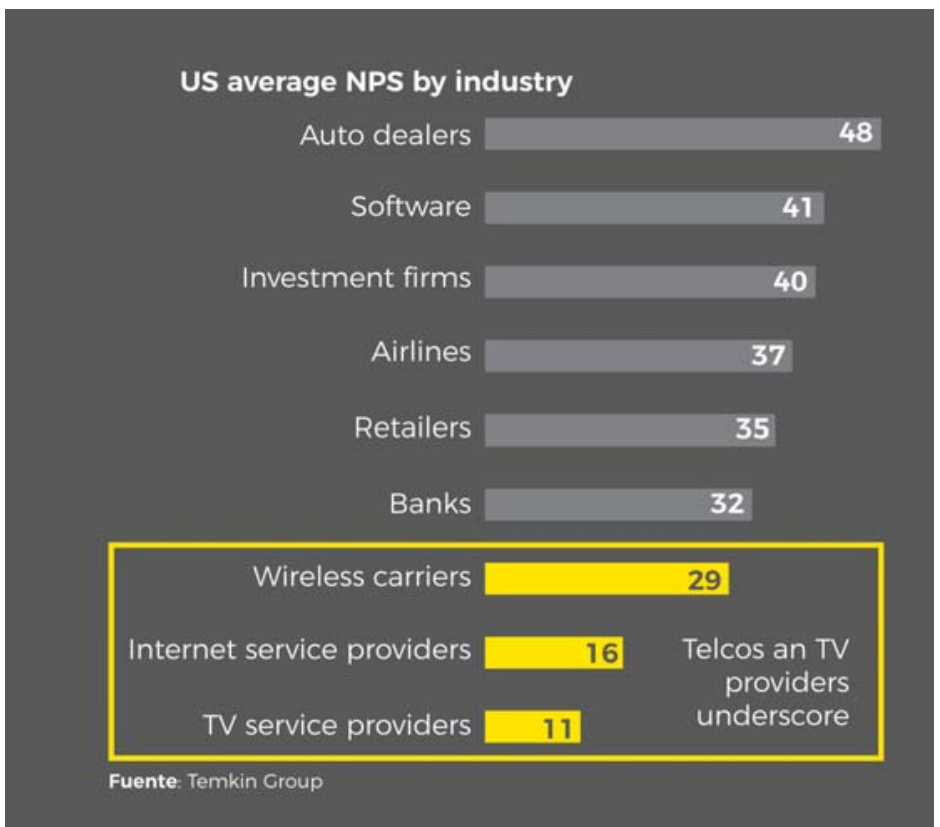

la innovación para los asistentes digitales y el mundo más amplio del loT.

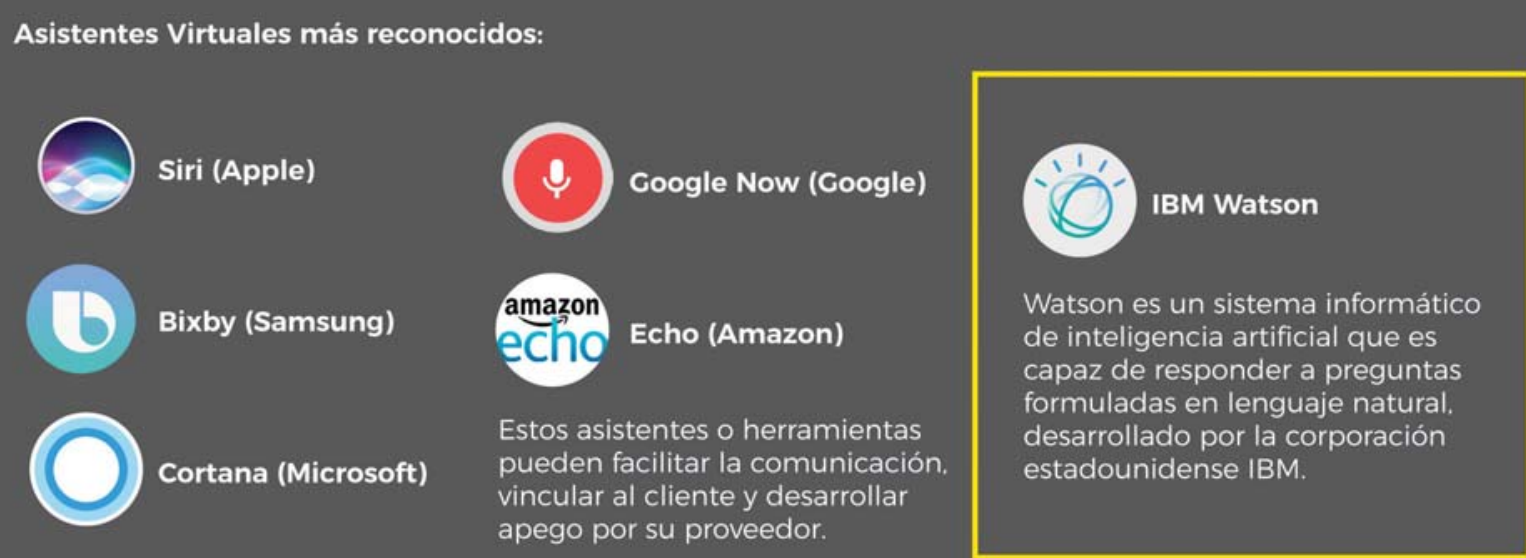




\title{
6 SEGURIDAD Y LA PRIVACIDAD
}

\author{
* EN CAMINO HACIA LA CONFIANZA
}

\section{CONTEXTO:}

- En un mundo de dispositivos conectados $24 / 7$ los usuarios empiezan a preocuparse acerca de la privacidad de sus datos.

- Los gigantes de tecnología y especialistas en ciberseguridad trabajan juntos para aumentar la concientización, asegurar los datos y proteger dispositivos.

- El Informe Ciberseguridad 2016 demuestra que en América Latina y el Caribe no estamos preparados para la economía digital.

\section{Aspectos destacados del informe:}

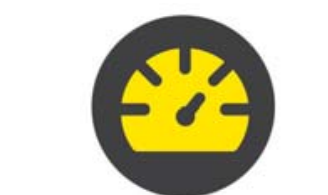

Revela que cuatro de cada cinco países de la región no tienen estrategias de ciberseguridad o planes de protección de infraestructura crítica. Dos de cada tres no cuentan con un centro de comando y control de seguridad cibernética. La gran mayoría de las fiscalías carece de capacidad para perseguir los delitos cibernéticos, entre otras carencias.

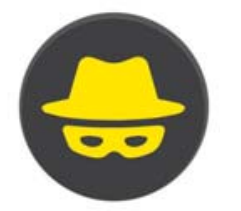

Los líderes del Banco Interamericano de Desarrollo (BID) y la Organización de los Estados Americanos (OEA) hicieron un llamado a los países de América Latina y el Caribe a acelerar sus esfuerzos en ciberseguridad.

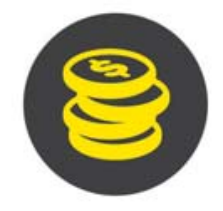

Gartner pronostica que el gasto mundial en seguridad de la información este año 2017 llegará a los 90.000 millones de dólares, un 7,6\% más que el año anterior, y calcula que esa cifra alcanzará los 113.000 millones de dólares en 2020.

\section{NUEVA TENDENCIA}

La capacidad creciente de los consumidores para recopilar, acceder y rediseñar sus propios datos ya le está dando vida a nuevos modelos de negocios estos 


\section{SIGUIENTES PASOS}

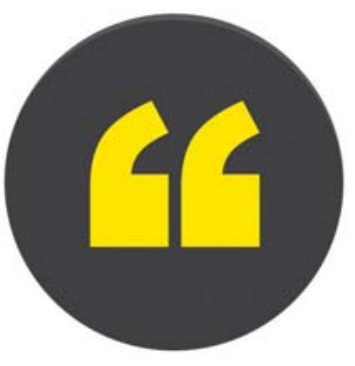

"La convergencia de nuestros sectores de Tecnología, Medios \& Entretenimiento y Telecomunicaciones, está bien encaminada, no sólo en EY, sino en el mercado, y estamos impulsando nuestra estrategia TMT en todo el mundo. Queremos ser líderes del mercado y el empleador de preferencia para los profesionales del área, pero requerimos asegurar que $t$ odos los profesionales de la firma estén conscientes e involucrados mientras avanzamos hacia una economía digital. Nuestro equipo de liderazgo espera una estrategia unificada y de escala global que satisfaga las necesidades los clientes."

INICIATIVAS DE EY GLOBAL

\section{- NUEVA ESTRATEGIA DE COMUNICACIONES INTERNAS DE TMT}

El contenido reflejará una estrecha colaboración entre el liderazgo de TMT, comunicaciones, conocimiento, marca y líneas de servicio para crear actualizaciones relevantes y atractivas que le ayudarán a hacer su trabajo y ofrecer valor a nuestros clientes.

\section{- LA NUEVA PÁGINA TMT}

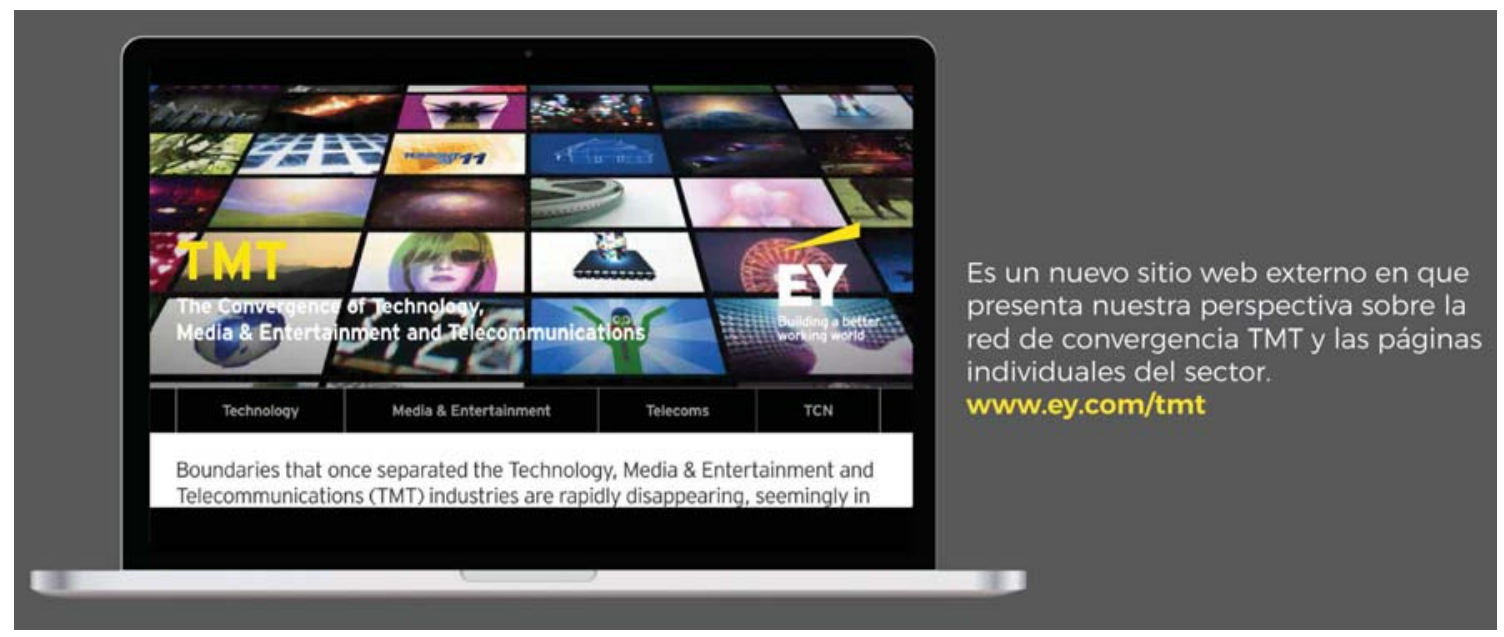




\section{ANEXO 1}

\begin{tabular}{|c|c|c|c|c|}
\hline Pais & Marca & Matriz & Tecnologia/Estándar & Banda / Espectro \\
\hline \multirow{5}{*}{ Costa Rica } & Kölbi & ICE & GSM/UMTS/HSDPA/LTE(4G) & $850 / 1800,1800,2600 \mathrm{MHz}$ \\
\hline & Claro & América Móvil & GSM/UMTS/HSDPA/LTE(4G) & $1800,1800 / 2100,1800 \mathrm{MHz}$ \\
\hline & Movistar & Telefónica & GSM/UMTS/HSDPA/LTE(4G) & $\begin{array}{c}850 / 1800,850 / 1800 / 2100 . \\
1800 \mathrm{MHz} \\
\end{array}$ \\
\hline & TuyoMóvil (OMV) & Televisora de Costa Rica & CSM/UMTS/HSDPA(3.5G) & $850 / 1800 \mathrm{MHz}$ \\
\hline & Fullmóvil (OMV) & Racsa & GSM/UMTS/HSDPA(3.5C) & $850 / 1800 \mathrm{MHz}$ \\
\hline \multirow{4}{*}{ El Salvador } & Tigo & Millicom & $\begin{array}{c}\text { AMPS/TDMA/CSM/GPRS/UMTS/ } \\
\text { HSPA+/LTE (4G) }\end{array}$ & 850/1900 MHz/850MHz (LTE) \\
\hline & Claro & América Móvil & CSM/UMTS/HSDPA/HSPA+(3.9C) & $1900 \mathrm{MHz}$ \\
\hline & Movistar & Telefónica & $\begin{array}{l}\text { CDMA/CSM/UMTS/HSPA+/LTE } \\
\text { (4G) }\end{array}$ & $850 / 1900 \mathrm{MHz} / 1900 \mathrm{MHz}$ (LTE) \\
\hline & Digicel & Digicel Group Limited & $\begin{array}{c}\text { GSM/GPRS/EDGE/HSDPA/ } \\
H S P A+(3.9 G)\end{array}$ & $900 / 1900 \mathrm{MHz}$ \\
\hline \multirow{5}{*}{ Guatemala } & Movistar & Telefónica & $\begin{array}{l}\text { CDMA/EV-DO/CSM/GPRS/EDCE/ } \\
\text { UMTS/HSDPA/HSPA+/LTE(4G) }\end{array}$ & $1900 \mathrm{MHz}$ \\
\hline & Tigo & Millicom & $\begin{array}{c}\text { GSM/GRPS/EDGE/UMTS/HSDPA } \\
\text { HSPA+Mi-MAX (4C) }\end{array}$ & $850 \mathrm{MHz}$ \\
\hline & Claro & América Móvil & $\begin{array}{c}\text { CDMA/CSM/CPRS/EDCE/UMTS/ } \\
\text { HSDPA/HSPA+(3.9C) }\end{array}$ & $900 / 1900 \mathrm{MHz}$ \\
\hline & Digicel & Digicel Group Limited & GSM/GPRS/EDCE(2.9G) & $900 \mathrm{MHz}$ \\
\hline & RED & INTELFON & $\operatorname{IDEN}(2 \mathrm{C})$ & $800 \mathrm{MHz}$ \\
\hline \multirow{2}{*}{ Honduras } & Tigo & Millicom & $\begin{array}{c}\text { AMPS/CDMA/CSM/CPRS/UMTS/ } \\
\text { HSDPA/HSPA+/LTE(4G) }\end{array}$ & $850.1700 / 2100 \mathrm{MHz}$ \\
\hline & Claro & América Móvil & GSM/UMTS/HSPA(3.8G) & $1900 \mathrm{MHz}$ \\
\hline \multirow{3}{*}{ Nicaragua } & Movistar & Telefónica & $\begin{array}{c}\text { TDMA/CDMA/CSM/UMTS/HSD } \\
\text { PA/HSPA/HSPA+/LTE(4G) }\end{array}$ & $850 / 1900 \mathrm{MHz}$ \\
\hline & Claro & América Móvil & $\begin{array}{c}\text { GSM/GPRS/EDGE/UMTS/HSDPA } \\
\text { HSPA/HSPA+/LTE(4G) }\end{array}$ & $850 / 1900 \mathrm{MHz}$ \\
\hline & Cootel & Xinwei & McWill & \\
\hline \multirow{4}{*}{ Panamá } & Movistar & Telefónica & $\begin{array}{c}\text { GSM/UMTS/HSDPA/HSPA/ } \\
\text { HSPA+/LTE(4G) }\end{array}$ & $\begin{array}{l}\text { 850/1900 MHz/700 MHz ATP } \\
\text { banda } 28\end{array}$ \\
\hline & Claro & América Móvil & $\begin{array}{c}\text { GSM/GPRS/EDGE/UMTS/HSDPA } \\
\text { HSPA+/LTE(4G) }\end{array}$ & $\begin{array}{c}1900 \mathrm{MHz} / 700 \mathrm{MHz} \text { ATP ban- } \\
\text { da } 28 / 1900 \mathrm{MHz} \text { banda } 2\end{array}$ \\
\hline & Digicel & Digicel Croup Limited & $\begin{array}{c}\text { GSM/GPRS/EDGE/HSDPA } \\
\text { HSPA+(3.9G) }\end{array}$ & $1900 \mathrm{MHz}$ \\
\hline & + Móvil & Cable \& Wireless Panamá & $\begin{array}{l}\text { GSM/UMTS/HSDPA/HSPA+/ } \\
\text { LTE(4G) }\end{array}$ & $\begin{array}{c}850 / 1900 \mathrm{MHz} / 700 \mathrm{MHz} \text { ATP } \\
\text { banda } 28\end{array}$ \\
\hline \multirow{4}{*}{$\begin{array}{l}\text { República } \\
\text { Dominicana }\end{array}$} & Claro & América Móvil & $\begin{array}{c}\text { CDMA/CSM/GPRS/EDGE/UMTS/ } \\
\text { HSDPA }\end{array}$ & $\begin{array}{c}850 \mathrm{MHz} / 1900 \mathrm{MHz} / 1700 \\
\mathrm{Mhz} / 2100 \mathrm{Mhz} \text { (Banda } 4 \\
\text { AWS) }\end{array}$ \\
\hline & Orange & $\begin{array}{c}\text { Orange } \\
\text { Telecomunicaciones } \\
\end{array}$ & GSM/GPRS/EDGE/UMTS/HSDPA & $\begin{array}{l}900 \mathrm{MHz} / 1800 \mathrm{MHz} / 1900 \\
\mathrm{MHz} / 1800 \mathrm{Mhz} \text { (Banda 3) }\end{array}$ \\
\hline & Tricom & Tricom & CDMANIMAX & $1900 \mathrm{MHz}$ \\
\hline & Viva & Trilogy Dominicana & CDMA/CSM/GPRS/EDGE & $\begin{array}{l}800 \mathrm{MHz} / 850 \mathrm{MHz} / 1900 \\
\mathrm{MHz} / 1900 \mathrm{MHz} \text { (Banda 2) }\end{array}$ \\
\hline
\end{tabular}

Tendencias Sector TMT | EY Centroamérica, Panamá y República Dominicana | Agosto 2017 


\section{EY | Assurance | Tax | Transactions | Consulting}

\section{Acerca de EY}

EY es un líder global en servicios de auditoría, impuestos,

transacciones y consultoría. Nuestro conocimiento, puntos de vista y servicios de calidad ayudan a generar confianza en los mercados de capital y en las economías alrededor del mundo. Desarrollamos líderes excepcionales que trabajan en equipo para cumplir las promesas hechas a todos nuestros grupos de interés. Al hacerlo, desempeñamos un papel fundamental en la construcción de un mejor mundo de negocios para nuestra gente, nuestros clientes y nuestras comunidades.

EY se refiere a la organización global de firmas miembro conocidas como Ernst \& Young Global Limited, en la que cada una de ellas actúa como una entidad legal separada. Ernst \& Young Global Limited, compañía del Reino Unido limitada por garantía, no provee servicios a clientes. Más información en www.ey.com.

() 2017 E\&Y Central America Inc.

Todos los derechos reservados.

Esta publicación contiene información en forma de resumen y, por lo tanto, su uso es solo para orientación general. No debe considerarse como sustituto de la investigación detallada o del ejercicio de un criterio profesional. Ni E\&Y Central America Inc., ni ningún otro miembro de la organización global de EY acepta responsabilidad alguna por la pérdida ocasionada a cualquier persona que actúe o deje de actuar como resultado de algún contenido en esta publicación. Sobre cualquier asunto en particular, referirse al asesor apropiado.

Los puntos de vista de terceros expuestos en la presente publicación no necesariamente son los puntos de vista de la organización global de EY o de sus firmas integrantes. Por ende, dichos puntos de vista se deben tomar en el contexto del momento en que se expresaron.

ey.com

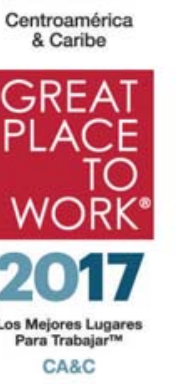

\section{(3) Contacto}

Carlos Gallegos

Líder del Sector Government

\& Public Sector

(+506) 2208-9800

carlos.gallegos@cr.ey.com

\section{Laureen Fernández}

Inteligencia de Negocios BD\&C (+506) 2208-6693

laureen.fernandez@cr.ey.com 